\title{
Body Mass and Risk
}

\author{
Norman K. Hollenberg
}

Published online: 5 May 2011

(C) Springer Science+Business Media, LLC 2011

Keywords Body mass index · BMI - Risk of death - Death from any cause - Asia - India - Bangladesh - China - Japan . Korea $\cdot$ Underweight $\cdot U$-shaped association

Zheng W, McLerran DF, Rolland B, et al.: Association between body-mass index and risk of death in more than 1 million Asians. $N$ Engl J Med 2011, 364:719-729.

\begin{abstract}
Background: Most studies that have evaluated the association between the body-mass index (BMI) and the risks of death from any cause and from specific causes have been conducted in populations of European origin.

Methods: We performed pooled analyses to evaluate the association between BMI and the risk of death among more than 1.1 million persons recruited in 19 cohorts in Asia. The analyses included approximately 120,700 deaths that occurred during a mean follow-up period of 9.2 years. Cox regression models were used to adjust for confounding factors.

Results: In the cohorts of East Asians, including Chinese, Japanese, and Koreans, the lowest risk of death was seen among persons with a BMI (the weight in kilograms divided by the square of the height in meters) in the range of 22.6 to 27.5. The risk was elevated among persons with BMI levels either higher or lower than that range-by a factor of up to 1.5 among those with a BMI of more than
\end{abstract}

\footnotetext{
N. K. Hollenberg $(\bowtie)$

Departments of Medicine and Radiology,

Brigham and Women's Hospital, Harvard Medical School,

Mailstop PBB1-Rad Building, 15 Francis Street,

Boston, MA 02115, USA

e-mail: djpagecapo@rics.bwh.harvard.edu
}

35.0 and by a factor of 2.8 among those with a BMI of 15.0 or less. A similar U-shaped association was seen between BMI and the risks of death from cancer, from cardiovascular diseases, and from other causes. In the cohorts comprising Indians and Bangladeshis, the risks of death from any cause and from causes other than cancer or cardiovascular disease were increased among persons with a BMI of 20.0 or less, as compared with those with a BMI of 22.6 to 25.0, whereas there was no excess risk of either death from any cause or cause-specific death associated with a high BMI.

Conclusions: Underweight was associated with a substantially increased risk of death in all Asian populations. The excess risk of death associated with a high BMI, however, was seen among East Asians but not among Indians and Bangladeshis.

\section{Editor's Comment}

There are substantially more than a million people in this analysis, and there were over 100,000 deaths over a mean follow-up of just about 10 years. What have we learned? In general, those with a high BMI carried a high risk, and those with a low BMI carried a low risk. That is not surprising. It is in the details that we learn new and powerful things. Underweight was associated with a substantial increase in death in all Asian populations. The excess risks associated with a high BMI, however, were seen among East Asians but not among Indians and Bangladeshis. Why this should be so is not clear, but it does provide room for substantial hypothesis generation.

Disclosure No potential conflicts of interest relevant to this article were reported. 